\title{
Relación entre calidad del sueño, ansiedad y depresión en pacientes con enfermedad renal crónica en hemodiálisis
}

\author{
Ana Vasco Gómez, Cristina Herrera Morales, Yaiza Martínez Delgado, Ernestina Junyent Iglesias, Guillermo \\ Pedreira Robles
}

Servicio de Nefrología del Hospital del Mar. Parc de Salut Mar. Barcelona. España

\section{Resumen}

Introducción: Los trastornos de sueño tienen una alta prevalencia en los pacientes con Enfermedad Renal Crónica en Hemodiálisis. Un descanso insuficiente o de mala calidad influye negativamente en la actividad cotidiana siendo causa o consecuencia de estados depresivos 0 ansiosos.

Objetivo: Evaluar la calidad del sueño en pacientes en hemodiálisis y su relación con los niveles de depresión y ansiedad.

Material y Método: Estudio observacional descriptivo con inclusión de 28 pacientes en hemodiálisis durante febrero de 2017. Se recogieron datos sociodemográficos, se administró el test Pittsburg de Calidad del Sueño, y se valoró el estado cognitivo con el examen Minimental y la depresión y ansiedad con la Hospital Anxiety and Depression Scale (HADS).

Resultados: El 82,14\% ( $n=23$ ) presentó insomnio según el test de Pittsburg. Los resultados obtenidos con el HADS fueron positivos para ansiedad en un $21 \%$ $(n=6)$ y positivos para depresión en un $35 \%(n=10)$. Los resultados en el test Pittsburg se correlacionaron positivamente con los niveles de ansiedad $(p=0,004)$ y depresión $(p=0,049)$. El grado de depresión se correlacionó negativamente con ausencia de deterioro cognitivo $(p=0,010)$.

\section{Correspondencia:}

Ana Vasco Gómez

Servicio Hemodiálisis Hospital del Mar

Passeig Marítim, 25-29. 08003 Barcelona

E-mail: avasco@parcdesalutmar.cat
Conclusiones: La calidad del sueño en los pacientes examinados es mala y se pueden relacionar con los resultados de depresión y ansiedad encontrados. Según estos resultados, incluiremos información sobre hábitos de sueño saludables en el proceso de educación a pacientes en hemodiálisis, así como pretendemos mejorar la detección y tratamiento especializado de la ansiedad y depresión.

PALABRAS CLAVE: calidad del sueño; insomnio; depresión; ansiedad; hemodiálisis.

\section{Relationship between sleep quality, anxiety and depression in patients with chronic kidney disease on hemodialysis}

\section{Abstract}

Introduction: Sleep disorders have a high prevalence in patients with chronic kidney disease (CKD) on hemodialysis (HD). Insufficient or poor quality rest negatively influences daily activity, being the cause or consequence of depressive or anxious states.

Objective: To evaluate the quality of sleep of patients on hemodialysis and the relationship with levels of depression and anxiety.

Material and Method: Descriptive observational study including 28 HD patients during February 2017. Sociodemographic data were collected, the Pittsburg Sleep Quality test was administered, the cognitive status was assessed with the Mini-Mental test and anxiety and depression was assessed with the Hospital Anxiety and Depression Scale (HADS). 
Results: According to the Pittsburg Sleep Quality test, $82.14 \%(n=23)$ presented insomnia. HADS was positively related to anxiety in $21 \%(n=6)$ of population and to depression in $35 \%(n=10)$. The results in the Pittsburgh test correlated positively with levels of anxiety $(p=0.004)$ and depression $(p=0.049)$. The degree of depression correlates negatively with the absence of cognitive impairment $(p=0.010)$.

Conclusions: The sleep quality in the examined patients is poor and can be related with the depression and anxiety results. According to that results, we will include information about healthy sleep habits in the HD patients' education process, as well as we intend to improve management and specialized treatment of anxiety and depression.

KEYWORDS: quality of sleep; insomnia; depression; anxiety; hemodialysis.

\section{Introducción}

El sueño es un estado activo en el que suceden modificaciones metabólicas, hormonales y bioquímicas necesarias para el buen funcionamiento del organismo. Todas las personas necesitan de un buen descanso para afrontar la actividad diaria en plenas condiciones tanto físicas como psíquicas ${ }^{1}$.

Los trastornos de sueño tienen una alta prevalencia en la población en general ${ }^{2}$ y se presentan con mayor frecuencia en los pacientes con Enfermedad Renal Crónica (ERC) en Hemodiálisis (HD) 2,3,4.

Los pacientes en $H D$, en muchas ocasiones, utilizan el tiempo de la sesión para dormir, interfiriendo en su patrón de sueño nocturno y por tanto en su descanso tan necesario. La carencia de sueño o la interrupción del ritmo circadiano aumenta el riesgo cardiovascular y, por ello, puede aumentar también la mortalidad en pacientes en hemodiálisis ${ }^{5,6}$. En general, no se percibe el insomnio como problema, son poco conocidas las consecuencias que puede provocar una mala calidad del sueño y el diagnóstico y tratamiento del insomnio queda muchas veces relegado7,8. Además, en muchas ocasiones, un descanso insuficiente o de mala calidad influye negativamente en la actividad cotidiana y es causa o consecuencia de estados depresivos y/0 ansiosos ${ }^{4,9,10}$.

Tanto la depresión como la ansiedad son trastornos habituales en los pacientes en $\mathrm{HD}^{11} \mathrm{y}$, de la misma manera que en la población en general, están infra tratados e infra diagnosticados ${ }^{7}$.

Por tanto el objetivo de nuestro trabajo ha sido evaluar la calidad del sueño en pacientes en HD y su relación con los niveles de depresión y ansiedad en la unidad de HD del servicio de nefrología del Hospital del Mar.

\section{Material y Método}

\section{Tipo de estudio}

Estudio observacional, descriptivo y de corte transversal.

\section{Muestra}

Se incluyeron un total de 28 pacientes en programa crónico de HD del Hospital del Mar de Barcelona durante febrero de 2017.

Como criterios de inclusión se establecieron: tener ERC, estar incluido en el programa de crónicos de HD del servicio, ser mayor de 18 años y firmar el consentimiento informado.

Como criterios de exclusión se establecieron no aceptar participar en el estudio y condiciones cognitivas severas o barrera idiomática que impedían contestar las escalas de valoración.

\section{Recogida de datos e instrumentos de medida}

Se recogieron datos sociodemográficos y clínicos (sexo, etiología de enfermedad renal, edad, peso, altura, IMC). También se recogieron datos de actividad física diaria de interés para relacionarlos con el descanso nocturno (ocupación laboral, actividad física diaria, participación en tareas domésticas y actividad sedentaria). Por otro lado, se administraron las escalas de valoración: Pittsburg, Minimental y Hospital Anxiety and Depression Scale, explicadas a continuación.

Para analizar el objetivo principal del estudio, se evaluaron los hábitos de sueño de los pacientes incluidos en el estudio, mediante un cuestionario ad hoc elaborado tras una revisión bibliográfica exhaustiva del tema a tratar (Anexo 1). Para lograr la validez del cuestionario diseñado, se examinó la claridad del lenguaje, la facilidad de uso, su relevancia para los profesionales y la idoneidad para los objetivos propuestos. 


\section{Cuestionario Pittsburg de Calidad del Sueño}

El índice de Calidad de Sueño de Pittsburgh (ICSP) es un cuestionario autoadministrado que mide la calidad de sueño y sus alteraciones en el último mes. Consta de 19 ítems autoevaluados por el paciente y 5 cuestiones evaluadas por el compañero/a de cama. Las cinco últimas cuestiones son utilizadas como información clínica, pero no contribuyen a la puntuación total del resultado. Los 19 ítems analizan los diferentes factores determinantes de la calidad del sueño, que se agrupan en 7 componentes: calidad del sueño, latencia del sueño, duración del sueño, eficiencia del sueño, alteraciones del sueño, uso de medicación para dormir y disfunción diurna. Cada componente se puntúa de 0 a 3 . De la suma de los 7 componentes se obtiene la puntuación total que oscila de 0 a 21 puntos, a mayor puntuación peor calidad de sueño $0^{12,13}$.

\section{Examen Minimental de estado cognitivo}

Test breve de cribado para el deterioro cognitivo. Incluye la evaluación de la orientación temporoespacial, la evocación inmediata, la atención y el cálculo, así como la evocación diferida, el lenguaje (en específico, denominación verbal, repetición, comprensión verbal, comprensión lectora, escritura) y la copia de una figura. La evaluación de los resultados transcurre entre la normalidad (27- 30 puntos), la sospecha patológica (25-26 puntos), el deterioro (12-24 puntos), la demencia (9-11 puntos) y la demencia grave $(0-8 \text { puntos })^{14}$.

Escala de ansiedad y depresión hospitalaria (HADS) Instrumento de autoevaluación para detectar el malestar emocional (depresión y ansiedad) en poblaciones con enfermedad física. Se compone de dos subescalas (HADA: ansiedad y HADD: depresión) de siete ítems cada una, en escala Likert de 0 a 3 y con rango de puntuación en cada subescala de 0 a 21 . A mayor puntuación en cada subescala, mayor ansiedad y depresión ${ }^{15}$.

\section{Análisis estadístico}

Se realizó un análisis descriptivo de las variables sociodemográficas, mostrando la distribución de frecuencias para las variables cualitativas y media y desviación estándar para las cuantitativas. Para el análisis de los datos, se utilizó la t de Student para la comparación de medias entre variables cuantitativas y la prueba Chi-cuadrado para la comparación de proporciones. Para poder establecer la correlación entre las variables estudiadas se ha utilizado el coeficiente de correlación lineal de Pearson ( $r$ ). El nivel de significación estadística se estableció en valores de $P$ inferiores a 0,05. EI análisis se realizó con el software estadístico SPSS 23 (IBM Corp.).

\section{Aspectos éticos}

Este proyecto se basa en los principios básicos que guían la atención y la investigación en el ámbito socio-sanitario: principio de autonomía o de respeto a las personas, principio de beneficencia, principio de justicia y principio de no maleficencia, recogidas en el Código de ética de las enfermeras de Cataluña y en la Declaración de Helsinki (Brasil, octubre 2013). A la misma vez, se respeta la confidencialidad de los datos obtenidos según la Ley Orgánica 15/1999 de Protección de Datos de Carácter Personal. Antes de la recogida de datos, se obtuvo el consentimiento informado firmado de todos los participantes.

\section{Resultados}

Se incluyeron en el estudio un total de 29 pacientes, excluyendo 1 paciente de la evaluación final por presentar criterios de demencia.

Las características sociodemográficas y clínicas de la muestra están representadas en la Tabla 1.

Tabla 1. Características sociodemográficas y clínicas de la muestra.

\begin{tabular}{|c|c|c|}
\hline \multirow[t]{2}{*}{ Sexo $(\% ; n)$} & Hombres & $64,28 \%(18)$ \\
\hline & Mujeres & $35,71 \%(10)$ \\
\hline \multirow[t]{6}{*}{ Enfermedad Renal (\%; n) } & No filiada & $50 \%(14)$ \\
\hline & DM & $17,86 \%(5)$ \\
\hline & HTA & $10,71 \%(3)$ \\
\hline & Glomerulares & $10,71 \%(3)$ \\
\hline & Vasculares & $7,14 \%(2)$ \\
\hline & Poliquísticas & $3,57 \%(1)$ \\
\hline Edad media (DE) & \multicolumn{2}{|l|}{$66,75 \pm 14,64$ aก̃os } \\
\hline Peso medio (DE) & \multicolumn{2}{|l|}{$68,85 \pm 17,76 \mathrm{~kg}$} \\
\hline Altura media (DE) & \multicolumn{2}{|l|}{$162,26 \pm 11,42 \mathrm{~cm}$} \\
\hline IMC medio (DE) & \multicolumn{2}{|l|}{$26,42 \pm 8,024$} \\
\hline \multirow[t]{2}{*}{ Turno de diálisis $(\% ; n)$} & Mañana & $64,29 \%(18)$ \\
\hline & Tarde & $35,71 \%(10)$ \\
\hline \multirow[t]{2}{*}{ Ocupación (\%; n) } & Laboralmente activo & $0 \%(0)$ \\
\hline & Laboralmente inactivo & $100 \%(28)$ \\
\hline
\end{tabular}

DM: diabetes mellitus, HTA: hipertensión arterial, DE: desviación estándar 
El $82,14 \%(n=23)$ presentaba algún grado de insomnio según el test de Pittsburg, siendo subclínico en un $64,29 \%$ ( $n=18$ ) y clínico en un $17,89 \%(n=5)$. La mediana del grupo fue de 11,07 [P25:8,75; P75:14] por lo que el grupo se clasifica en la categoría de insomnio subclínico (ver Figura 1).

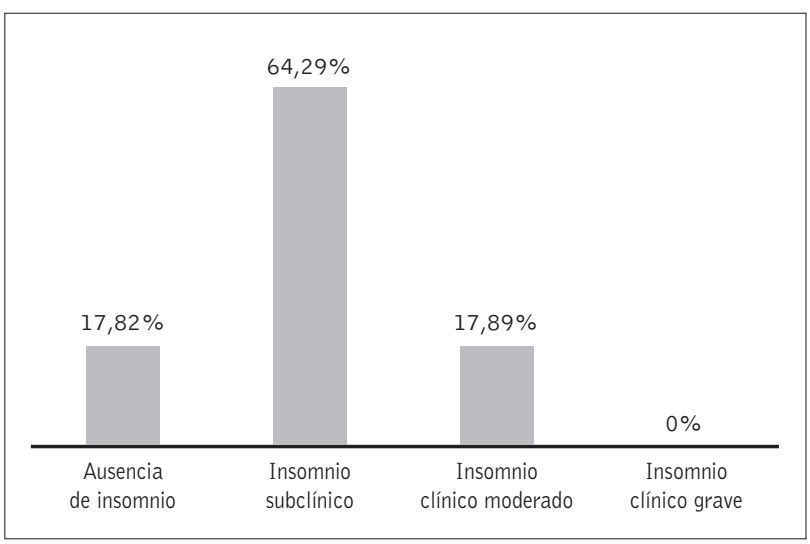

Figura 1. Resultados del Cuestionario Pittsburg de Calidad de Sueño.

Los valores obtenidos con el examen minimental son de un $75 \%(n=21)$ dentro de la normalidad. El $10,71 \%$ $(n=3)$ obtuvo parámetros de sospecha patológica y el $14,29 \%(n=4)$ de deterioro. Tan solo un 3,57\% ( $n=1)$ presentaba valores de demencia y fue excluido de las demás valoraciones y del estudio.

Los resultados obtenidos con el HADS fueron positivos para ansiedad en un $21,43 \%(n=6)$ y positivos para depresión en un $35,71 \%(n=10)$.

Los resultados del cuestionario Ad-hoc elaborado para profundizar en el análisis del posible insomnio de los pacientes evaluados se representan en la Tabla 2.

Los resultados en el test Pittsburg se correlacionaron positivamente con los niveles de ansiedad $(p=0,04)$ y depresión $(p=0,049)$. El grado de depresión se correlacionó negativamente con ausencia de deterioro cognitivo $(p=0,010)$. No se encontró ninguna otra relación significativa en el resto de parámetros recogidos ni en las respuesta del cuestionario ad hoc.

\section{Discusión}

La premisa inicial del estudio era que las personas con ERC habitualmente presentan trastornos en el patrón del sueño. La bibliografía consultada habla de has- ta un $60 \%$ de pacientes con problemas en su descanso $0^{2,4,16,18}$. En este estudio destaca el elevado porcentaje $(82,14 \%)$ de pacientes que perciben un mal descanso nocturno, según los resultados encontrados en el cuestionario de Pittsburg.

La presencia de alteraciones en el patrón de sueño con un porcentaje similar se ha encontrado en otros pacientes diagnosticados de depresión y/o ansiedad. Varios autores, como Cano-Lozano ${ }^{9}$, hablan de hasta un $80 \%$ de pacientes afectados por una disminución de la calidad y la cantidad de sueño en personas diagnosticadas de trastornos depresivos $y / 0$ ansiosos. Por este motivo es interesante destacar la correlación positiva obtenida en la muestra seleccionada entre los niveles de ansiedad y depresión y los resultados del test de Pittsburgh. En este punto, es difícil discernir, y así la bibliografia lo expone también, si los estados de ansiedad y depresión provocan problemas de sueño o es la incapacidad para conseguir un correcto descanso lo que inicia la aparición de los trastornos analizados.

De la misma manera que en el presente estudio, los problemas con la calidad del sueño se han relacionado previamente con trastornos como la ansiedad y la depresión en la población general ${ }^{16,18}$ así como en pacientes con dolor crónico como la fibromialgia ${ }^{18,19}$. Aunque cuando hablamos de ERC la mayoría de las publicaciones se centran en explorar como posibles causas del insomnio factores físicos como las piernas inquietas ${ }^{8,20}$, el prurito ${ }^{21}$, o el Sín-

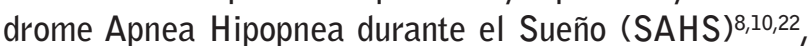
encontramos algún estudio ${ }^{2,22}$ que comparte con los resultados presentados en este estudio: la relación positiva entre el insomnio, la depresión y la ansiedad.

En cuanto a los factores demográficos como la edad y el sexo, varios estudios como el de López ${ }^{7}$, describen que, en la población general, la calidad del sueño disminuye con la edad y que existen diferencias entre los dos sexos. En los pacientes en HD, los resultados recogidos coinciden con otras publicaciones ${ }^{2,21,23}$ que analizan calidad del sueño en los pacientes de HD, donde no existe una relación significativa entre factores demográficos como la edad, el sexo y la calidad del sueño.

En los datos recogidos en la muestra explorada, la mayoría de los pacientes que verbalizan tener insomnio pertenecen al turno mañana $(64,29 \%)$. Tal y como ya describía Álvarez ${ }^{4}$, existen diferencias significativas relacionadas con los hábitos de sueño y el turno de diálisis, siendo el turno mañana determinante para la aparición de problemas de sueño. 
Tabla 2. Cuestionario $A d$ Hoc de valoración del patrón del sueño en la muestra.

\begin{tabular}{|c|c|c|}
\hline \multirow[t]{2}{*}{ ¿Toma fármacos para dormir? $(\% ; n)$} & Si & $39,28 \%(11)$ \\
\hline & No & $60,71 \%(17)$ \\
\hline \multirow[t]{5}{*}{ Nombre del fármaco que toma $(\% ; n)$} & Lormetazapam & $17,89 \%(5)$ \\
\hline & Clometiazol & $7,14 \%(2)$ \\
\hline & Valeriana & $3,57 \%(1)$ \\
\hline & Lorazepam & $10,71 \%(3)$ \\
\hline & No procede & $60,71(17)$ \\
\hline \multirow[t]{4}{*}{ ¿Por qué especialidad está prescrito? $(\% ; n)$} & Nefrología & $14,29 \%(4)$ \\
\hline & Psiquiatría & $14,29 \%(4)$ \\
\hline & Atención primaria & $10,71 \%(3)$ \\
\hline & No procede & $60,71(17)$ \\
\hline \multirow{2}{*}{$\begin{array}{l}\text { ¿Toma más de } 5 \text { vasos o tazas de té, café, } \\
\text { mate o bebidas cafeinadas? }(\% ; n)\end{array}$} & $\mathrm{Si}$ & $0 \%(0)$ \\
\hline & No & $100 \%(28)$ \\
\hline Horas (mediana) de sueño nocturnas & \multicolumn{2}{|c|}{6,42 horas [P25 5; P75 8] } \\
\hline Minutos (mediana) de sueño diurno & \multicolumn{2}{|c|}{60,35 minutos [P25 0; P75 105] } \\
\hline \multirow{2}{*}{$\begin{array}{l}\text { ¿Las horas de sueño nocturnas y diurnas cambian } \\
\text { en función del día de hemodi? }(\% ; n)\end{array}$} & $\mathrm{Si}$ & $46,42 \%(13)$ \\
\hline & No & $53,57 \%(15)$ \\
\hline $\begin{array}{l}\text { Horas (mediana) que el paciente cree que } \\
\text { debería dormir cada noche }\end{array}$ & \multicolumn{2}{|c|}{7,46 horas $\left[P_{25} 7 ; P_{75} 8\right]$} \\
\hline Tiempo (mediana) en cama antes de dormirse & \multicolumn{2}{|c|}{63,21 minutos $\left[P_{25} 30 ; P_{75} 62.41\right]$} \\
\hline Tiempo (mediana) en cama antes de levantarse & \multicolumn{2}{|c|}{51,63 minutos $\left[P_{25} 0 ; P_{75} 76.54\right]$} \\
\hline \multirow[t]{2}{*}{ ¿Realiza actividades que induzcan el sueño? $(\% ; n)$} & Si & $17,89 \%(5)$ \\
\hline & No & $82,14 \%(23)$ \\
\hline \multirow[t]{2}{*}{ ¿Cree que tiene problemas para dormir?(\%;n) } & $\mathrm{Si}$ & $42,86 \%(12)$ \\
\hline & No & $57,14 \%(16)$ \\
\hline \multirow[t]{3}{*}{ ¿Empezaron por algún motivo los problemas para dormir?(\%; n) } & Si & $10,71 \%(3)$ \\
\hline & No & $32,14 \%(9)$ \\
\hline & No procede & $57,14 \%(16)$ \\
\hline \multirow[t]{2}{*}{ ¿Lo considera un problema importante?(\%; n) } & $\mathrm{Si}$ & $67,86 \%(19)$ \\
\hline & No & $32,14 \%(9)$ \\
\hline \multirow{2}{*}{$\begin{array}{l}\text { ¿Tiene problemas como ronquido intenso pausas respiratorias, } \\
\text { movimientos mientras duerme y/ohablar dormido? }(\% ; n)\end{array}$} & $\mathrm{Si}$ & $64,29 \%(18)$ \\
\hline & No & $35,71 \%(10)$ \\
\hline \multirow[t]{3}{*}{ Actividad física diaria $(\% ; n)$} & No realiza & $57,14 \%(16)$ \\
\hline & $\leq 30 \mathrm{~min}$ & $21,42 \%(6)$ \\
\hline & $>30 \min$ & $21,42 \%(6)$ \\
\hline \multirow[t]{3}{*}{ Participación en tareas domésticas (\%; n) } & No realiza & $60,71 \%(17)$ \\
\hline & Participa & $28,57 \%(8)$ \\
\hline & Las hace todas & $10,71 \%(3)$ \\
\hline \multirow[t]{3}{*}{ Actividad sedentaria $(\% ; n)$} & Cada día & $78,57 \%(22)$ \\
\hline & 1 hora cada 2-3 días & $7,14 \%(2)$ \\
\hline & Casi nunca & $14,28 \%(4)$ \\
\hline
\end{tabular}


Del $82,14 \%$ de pacientes que presentan alteraciones en su patrón de descanso nocturno según los resultados del cuestionario de Pittsburg, solo un $36 \%$ tiene medicación prescrita. Esto es entendible sabiendo que solo el $42 \%$ de los pacientes con problemas de sueño lo percibe como un problema. Referencias consultadas en relación con el tema apuntan a que intervenciones concretas por parte del personal sanitario pueden favorecer la detección y descubrimiento del problema y, a la misma vez, permiten mejorar los resultados encontrados. De esta manera, es interesante incluir la valoración de este campo en las valoraciones habituales de enfermería e incluir información sobre estos aspectos en la rutina educativa a los pacientes ${ }^{24}$.

En base a nuestros resultados podemos concluir que la mayoría de los pacientes encuestados tiene una mala calidad de sueño, aunque no lo considera un problema. Este hecho provoca que no se trate debidamente. Por otro lado, habiendo constatado la relación existente entre los niveles de depresión y ansiedad que presentan los pacientes con el insomnio, es igual de importante, la necesidad de mejorar la detección y el tratamiento especializado de la ansiedad y depresión.

Recibido: 26 diciembre 2017

Revisado: 2 febrero 2018

Modificado: 7 agosto 2018

Aceptado: 15 septiembre 2018

\section{Bibliografía}

1. Merino Fernández-Pellón A. Trastornos del sueño. Medicine. 2007;9(86):5550-72.

2. Pérez A. Factores relacionados con los trastornos del sueño en hemodiálisis. Diálisis y trasplante. 2004;25(1):27-32.

3. Peña Martínez B, Navarro V, Oshiro H, Bernabe Ortiz $A$. Factores asociados a mala calidad de sueño en pacientes con insuficiencia renal crónica en hemodiálisis. Diálisis y Trasplante. 2015;36(1):20-6.
4. Álvarez Ude F, Alamo C, Fernández Reyes M, Bravo $B$, Vicente $E$, Ferrer $M$, et al. Alteraciones del sueño y salud percibida en pacientes en hemodiálisis crónica. Rev Nefrología. 1999;19(2):168-76.

5. Masuda T, Murata M, Honma S, Iwazu Y, Sasaki N, Ogura M, et al. Sleep-disordered breathing predicts cardiovascular events and mortality in hemodialysis patients. Nephrol Dial Transplant. 2011;26:228995.

6. Zoccali C, Mallamaci F, Tripepi G. Nocturnal hypoxemia predicts incident cardiovascular complications in dialysis patients. J Am Soc Nephrol. 2002;13: 729-33.

7. López $F$, Fernández 0 , Fernández L, Mareque MA, Alejandre G, Báez J. Valoración Clínica y calidad de vida relacionada con la salud del paciente con insomnio. Rev Clín Med Fam. 2011;4(2):92-99.

8. Restrepo D, Cardeño Carlos A. Trastornos de sueño en los pacientes en diálisis. Rev Colombiana Psiquiatría. 2010; 39(3):588-600.

9. Cano Lozano MC, Espinosa Fernández L, Miró E, BuelaCasal G. Una revisión de las alteraciones del sueño en la depresión. Rev Neurol. 2003;36(4): 366-75.

10. Jurado Gámez B, Fernández Marín MC, Cobos MJ, García Gil F, Feu Collado F, Muñoz Cabrera L, Cosano Povedano A. Trastorno del sueño en pacientes en hemodiálisis. Estudio polisomnográfico con grupo control. Neumosur. 2007;19(4):171-78.

11. Pedreira Robles G, Vasco Gómez A, Herrera Morales $C$, Martínez Delgado $Y$, Junyent Iglesias $E$. Análisis del estado psicofísico de los pacientes en hemodiálisis. Enferm Nefrol. 2018;21(1):44-51

12. Lomelí $\mathrm{H} \mathrm{A}$, et al. Escalas y cuestionarios para evaluar el sueño: una revisión. Actas Esp Psiquiatr. 2008; 36(1):50-9.

13. Luna Y, Robles $Y$, Agüero $Y$, Validación del Índice de Calidad de Sueño de Pittsburgh en una Muestra Peruana. Anales salud Mental. 2015;21(2):23-30.

14. López J, Martí G. Mini-Examen Cognoscitivo (MEC). Rev Esp Med Legal. 2011;37(3):122-27. 
15. Terol-Cantero MC, Cabrera-Perona V, Martín-Aragón M. Revisión de estudios de la Escala de Ansiedad y Depresión Hospitalaria (HAD) en muestras españolas. Anales de Psico. 2015;31(2):494-503.

16. Miró E, Cano Lozano MC, Buela Casal G. Sueño y calidad de vida. Revista Colombiana de Psicología. 2005;14:11-27.

17. Vela-Bueno A, Iceta M, Fernandez C. Prevalencia de los trastornos del sueño en la ciudad de Madrid. Gac Sanit. 1999;13(6):441-8.

18. Manez, I, Fenellosa, P, Martínez A, Salazar A. Calidad del sueño, dolor y depresión en fibromialgia. Rev Soc Esp Dolor. 2005;12(8):491-500.

19. Velázquez I, Muñoz M, Sánchez M, Zenner A, Velázquez L. Trastornos del sueño e incidencia de ansiedad y depresión en pacientes con dolor crónico no maligno tratados con opioides potentes. Rev Soc Esp Dolor. 2012;19(2):49-58.
20. Restrepo Bernal $D$, Hidalgo Martínez $P$, Gómez-Restrepo C, Gil Laverde F, Cardeño Castro C. Trastornos del sueño en pacientes en hemodiálisis, prevalencia y características clínicas y epidemiológicas. Rev Colomb Psiquiat. 2011;40(3):433-45.

21. Giera L, Rica A, Martínez N, Sánchez F, Peña I. Evaluación de la Calidad del sueño en pacientes en hemodiálisis. Rev Enf Nefrol 2015;18(1):52-3.

22. Barrios $S$, Masalan $P$, De la Fuente $L$. Sueño y sus características en personas con trasplante renal. Rev Enf Nefrol. 2016;19(3):191-200.

23. López JM, Molina M, Fernández A. Análisis del patrón del sueño en pacientes en hemodiálisis. Rev Enfer Nefrol. 2014;17(1):118.

24. Soleimani $F$, Motaarefi $H$, Hasanpour-Dehkordi A. Effect of sleep hygiene education on sleep quality in hemodialysis patients. Journal of Clinical and Diagnostic Research. 2016;10(12):LC01-LC04. 


\section{Anexos}

Anexo 1. Cuestionario ad hoc de evaluación del patrón del sueño.

Paciente:

Peso:

Altura:

IMC:

¿Toma fármacos para dormir? $\square$ No $\square \mathrm{Si}$ :

$\square$ Hipnóticos $\square$ Antidepresivos $\square$ Sedantes

$\square$ Relajantes

Nombre del fármaco que toma, dosis y hora en que lo toma:

¿Por qué especialidad está prescrito?

Horas de sueño nocturnas:

Horas de sueño diurnas:

¿Las horas de sueño nocturnas y diurnas cambian en función del día de HD?

$\square$ SI $\square$ No

Si es que si, ¿cómo cambia?:

Horas que cree que debería dormir cada noche:

Tiempo en cama antes de dormirse:

Tiempo en cama antes de levantarse:

¿Cree que tiene problemas para dormir?

$\square \mathrm{Si}$

$\square$ No

¿Empezaron por algún motivo los problemas para dormir?

$\square \mathrm{Si}:$

¿Lo considera un problema importante?

$\square \mathrm{Si}$

$\square$ No

Actividad diaria:

$\square$ Ocupado laboralmente

$\square$ Desocupado laboralmente

$\square$ No realiza actividad física

$\square$ Realiza $30 \mathrm{~min} /$ día de actividad física

$\square$ Realiza más de $30 \mathrm{~min} /$ día de actividad física

$\square$ No realiza tareas domésticas $\square$ Participa en las tareas domesticas

$\square$ Realiza todas las tareas domesticas

$\square$ Casi nunca hace actividades sedentarias (TV+sofá, sentarse en el bar, echar siestas...)

$\square 2$ - 3 días/semana hace actividades sedentarias

$\square$ Cada día hace actividades sedentarias

Síntomas:

$\begin{array}{lll}\square \text { Disnea } & \square \text { Prurito } \quad \square \text { Nicturia } & \square \text { Piernas inquietas } \square \text { SAOS + CPAP } \\ \square \text { Dolor } & \square \text { Calambres musculares } & \square \text { Preocupaciones/nervios }\end{array}$

Este artículo se distribuye bajo una Licencia Creative Commons Atribución-NoComercial 4.0 Internacional. https://creativecommons.org/licenses/by-nc/4.0/

Open Access (c) (7) (8) 